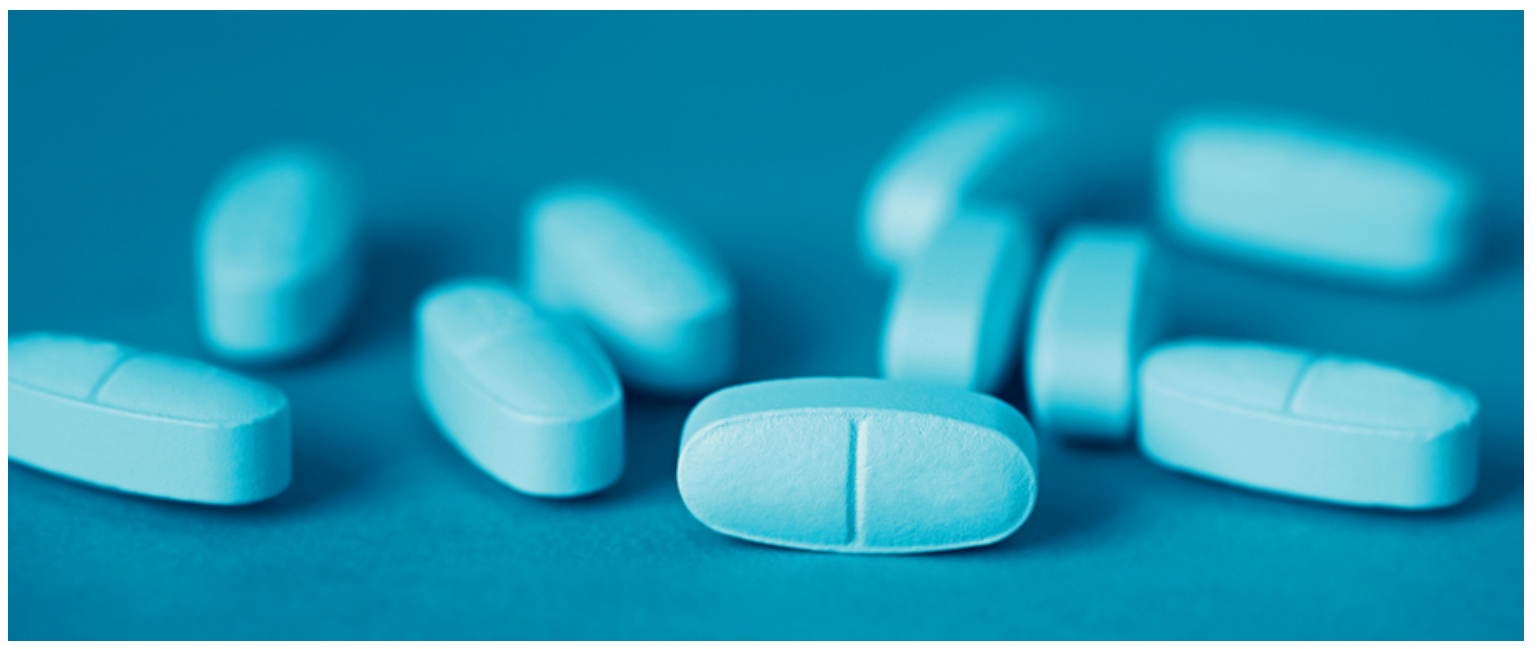

\title{
Protection de la bonne foi de l'assuré
}

\section{Valérie Junod}

Professeure de droit aux Universités de Genève et de Lausanne

'auteur remercie Dr Simon Olivier (Section d'addictologie, Service de psychiatrie communautaire, CHUV) pour ses observations et commentaires.
Le remboursement par l'assurance-maladie des médicaments est un thème délicat, sur le plan tant social que juridique. Les patients tiennent à ce que leurs médicaments soient pris en charge - rapidement et sans tracasserie administrative. Les assureurs cherchent à déceler les prescriptions inefficaces ou excessivement coûteuses. Les médecins sont parfois pris entre deux feux: à savoir le patient qui insiste pour recevoir son traitement et l'assurance qui menace de ne pas rembourser.

Un arrêt du Tribunal fédéral du 28 février 2017 met en évidence ce conflit ${ }^{1}$. La patiente souffrait depuis plusieurs années d'un trouble somatoforme douloureux ${ }^{2}$, trouble attribué à deux accidents survenus en 1997 et en 2000. Sans qu'on sache vraiment pourquoi ou comment, son médecin lui avait prescrit de la buprénorphine et de la morphine (deux formes d'opioïdes). Ces médicaments n'étaient pas venus à bout de ses douleurs et la patiente en était devenue (physiquement) dépendante. Sa caisse-maladie, qui avait pris en charge le traitement (à la suite de l'assurance-accident), avait d'abord assumé leur coût, puis en octobre 2014, avait déclaré refuser tout paiement, exigeant de surcroît le remboursement des montants précédemment payés. La caisse fondait sa décision sur le caractère en l'espèce non-efficace, non-adéquat et non-économique des deux médicaments.

\section{Points-clés de l'arrêt}

Sur recours de la patiente, le Tribunal fédéral confirme que les médicaments ne sont plus à charge de la caissemaladie, mais nie l'obligation faite à la patiente de rembourser les montants précédemment reçus.

S'agissant du premier aspect, le Tribunal commence par souligner que les deux médicaments sont inscrits sur la Liste des spécialités (LS; liste des médicaments à charge des caisses) tenue par l'Office fédéral de la santé publique (OFSP); ils ont été prescrits par un médecin conformément à leur indication médicale autorisée par l'Institut suisse des produits thérapeutiques, Swissmedic ${ }^{3}$. Les conditions fixées par la législation (Loi fédérale sur l'assurance-maladie (LAMal) et ses ordonnances) étant remplies, les médicaments sont en principe à charge de l'assurance obligatoire de soins (AOS). Le fait que ces médicaments sont susceptibles d'entraîner une dépendance physique ${ }^{4}$, voire un syndrome de dépendance ${ }^{5}$, le fait qu'ils ne guérissent pas la douleur et le fait que des études récentes ont mis en doute leur utilité dans le traitement de la douleur somatoforme n'y changent rien. En effet, tant que l'OFSP ne modifie pas l'inscription dans la LS, par exemple en y adjoignant une limitation (i.e., une condition supplémentaire pour le remboursement), l'inscription a une portée contraignante pour les caisses ${ }^{6}$. 
Le Tribunal fédéral opère cependant une distinction entre l'efficacité et l'adéquation appréciées au début du traitement et celles appréciées lors de la poursuite du traitement à long terme. Dans le cas présent, il considère que la patiente avait une prétention légitime à la prise en charge de ses deux médicaments au début du traitement, mais que le problème se pose différemment lors de sa continuation. En effet, différents rapports médicaux avaient conclu que le traitement par opioïde était devenu nuisible pour la santé de la patiente, sa dépendance physique étant patente; par ailleurs, les opioïdes prescrits ne parvenaient même plus à éliminer ses douleurs. Dans ces conditions, le Tribunal fédéral conclut que les exigences légales d'efficacité, d'adéquation et d'économicité ont cessé d'être remplies, avec pour conséquence que les médicaments ne sont plus à charge de l'AOS.

La deuxième partie de l'arrêt s'interroge sur le moment à partir duquel le traitement a cessé d'être à charge et donc du moment à partir duquel la patiente est éventuellement tenue au remboursement. Le Tribunal fédéral retient que la patiente ne peut se voir imposer le remboursement pour la période antérieure au premier courrier de la caisse par lequel celle-ci annonçait mettre fin à ses prestations. Cette décision de la caisse remontant au $1^{\mathrm{er}}$ octobre 2014 , les paiements opérés avant cette date ne peuvent être remis en cause, et ce en dépit du constat médical, déjà en 2011, d'une dépendance avérée $^{7}$. Ainsi, le Tribunal fait primer la protection de la confiance de la patiente envers la caisse (en termes juridiques: droit à la protection de la bonne foi en lien avec les renseignements fournis par une autorité) ${ }^{8}$. Formulé différemment, la patiente qui recevait ses médicaments sur la base d'une prescription de son médecin et qui, dans le passé, en avait obtenu régulièrement la prise en charge par la caisse n'avait - jusqu'au $1^{\text {er }}$ octobre 2014 pas de raison de soupçonner que la caisse reviendrait sur sa pratique; ce n'est qu'à partir dudit courrier du $1^{\mathrm{er}}$ octobre 2014 que la patiente est avertie et ne peut donc plus invoquer la protection de sa bonne foi. Cependant, le Tribunal rappelle sa jurisprudence exigeant qu'un délai transitoire approprié soit octroyé à l'assuré lorsqu'un traitement à long terme doit être modifié. C'est tout particulièrement le cas s'agissant d'opioïdes ayant provoqué une dépendance physique: la réduction des doses doit impérativement se faire progressivement. Le Tribunal fédéral renvoie l'affaire à la caisse pour qu'elle détermine à quel rythme les doses doivent être diminuées et les montants à payer qui en résultent pour elle.

\section{Commentaires et critique}

Trois aspects de l'arrêt méritent d'être mis en évidence. Tout d'abord, au moment où une thérapie est débutée, il est rassurant pour les patients de savoir que sa prise en charge est garantie du moment que le médicament figure sur la LS et qu'il a été prescrit conformément à son indication thérapeutique. Les patients n'ont pas à se soucier d'études ou d'articles scientifiques remettant en cause l'efficacité ou l'adéquation du traitement tant que ces connaissances n'ont pas été «intégrées» dans la notice d'emploi émise par Swissmedic et/ou dans la LS de l'OFSP. Ce résultat est équitable car les patients - comme leurs médecins d'ailleurs - ne peuvent se tenir au courant de toutes les nouvelles études; s'agissant en tout cas de la prise en charge par l'AOS, ils doivent pouvoir se fier aux décisions prises par les deux autorités susvisées.

Deuxième point important: l'arrêt révèle que cette position de principe - la foi en l'AMM et la LS - ne vaut plus nécessairement lorsque doit être décidée la poursuite de la thérapie à charge de l'AOS. A ce moment, il faut au contraire évaluer si le médicament prescrit est bel et bien efficace et adéquat pour le patient en cause. Dans notre affaire, le cas était facile à trancher, car les médecins s'accordaient pour dire que la patiente était devenue dépendante aux opioïdes sans que ses douleurs n'en soient pour autant supprimées; l'arrêt ne mentionne aucune circonstance qui aurait pu jeter le doute sur ce constat médical.

Toutefois, les autres affaires que les caisses auront à trancher risquent d'être plus complexes. D'où des questions que le Tribunal ne tranche pas, notamment le moment à partir duquel il peut être exigé une preuve que le médicament est bel et bien efficace dans le cas du patient particulier ainsi que la nature et la fiabilité de cette preuve ${ }^{9}$. Le Tribunal fédéral dit bien qu'on ne peut exiger qu'un médicament guérisse le patient dans tous les cas et que le soulagement de certains symptômes de la maladie peut parfaitement suffire. Reste que, dans les litiges avec les assureurs, la question de savoir quel rapport bénéfice-risque peut être exigé est difficile.

Troisième point important qui vient d'ailleurs contrebalancer le précédent: la protection de la bonne foi dans les rapports assuré-assureur. Tant que l'assureur n'a pas signalé qu'il refuse (pour l'avenir) la prise en charge, le patient peut légitimement s'attendre à ce que son médicament, prescrit par un médecin conformément tant à son indication qu'à la LS et antérieurement payé par la caisse, continue à l'être ${ }^{10}$. Sa bonne foi est protégée même si des expertises médicales ont conclu à l'inefficacité du médicament! Sur cet aspect, le Tribunal se montre généreux, plaçant la responsabilité d'agir sur les seules caisses. Ceci est légitime car, de nouveau, les assurés ne sont guère à même de mettre en doute les choix thérapeutiques de leur médecin, ni d'anticiper les 
réactions de leur caisse ${ }^{11}$. Qu'il suffise à une caisse de régler sans objection quelques factures (ici une douzaine pendant 7 mois) pour susciter la confiance des assurés quant au caractère définitif de ces paiements renforce significativement les droits de ces derniers.

En revanche, l'arrêt pourrait amener les caisses à intervenir plus rapidement lorsqu'elles soupçonnent qu'un traitement ne remplit plus les conditions de la LAMal. En effet, à partir du moment où elles communiquent à leur assuré leur refus d'assumer la prise en charge, elles «neutralisent» les effets de la confiance et - pour autant bien sûr que leur prise de position soit confirmée par la justice en cas de recours - mettent fin à leur obligation de paiement.

\section{Conclusion}

S'agissant du syndrome de dépendance aux opioïdes, le Tribunal relève que le risque de dépendance est intrinsèque à l'usage des médicaments opioïdes, sans que cela ne remette en cause la pertinence du traitement de la douleur. L'arrêt rappelle aussi que le sevrage de tels médicaments (lorsque leur usage cesse d'être indiqué) doit intervenir progressivement, et non pas brutalement comme l'entendait la caisse. Ces deux aspects sont à saluer.

Cependant, le lecteur reste quelque peu "perplexe» en ce qui concerne la relation unissant la patiente à son médecin. En effet, celui-ci a poursuivi pendant des années un traitement qui, non seulement ne parvenait pas à juguler les douleurs de la patiente, mais a provoqué chez elle une dépendance aux opioïdes. Pourquoi, face à ce constat, ne s'est-il pas interrogé sur l'existence d'un syndrome de dépendance iatrogène aux opioïdes? A supposer ce diagnostic correct, le médecin aurait pu sélectionner un médicament comme la méthadone, la buprénorphine ou la morphine-retard afin précisément d'introduire un traitement dit «agoniste aux opioïdes» ou TAO (historiquement et encore couramment désigné comme "traitement basé sur la substitution»). La caisse aurait alors été tenue de prendre en charge le TAO, et ce même à long terme; pour sa part, la patiente aurait été amenée à faire un choix explicite quant aux modalités du traitement de sa dépendance; quant au médecin, il aurait été à même de proposer un traitement pour lequel les preuves d'efficacité, d'adéquation et d'économicité sont incontestables. Certes, là n'était pas la question juridique soumise au Tribunal, mais on peut néanmoins se demander pourquoi cette «échappatoire» élégante n’a pas été envisagée.

\section{Crédit photo}

(c) Robynmac | Dreamstime.com
Notes de bas de page

1 Arrêt 9C_528/2016 du 28 février 2016, prévu pour publication dans la collection des ATF (arrêts du Tribunal fédéral). Pour une meilleure compréhension des faits, le lecteur peut se référer à un précédent litige opposant la même patiente cette fois-ci à son assureur-invalidité (cf. arrêt 8C_545/2013 du 12 novembre 2013).

2 Selon la classification internationale des maladies (CIM 10) de l'Organisation mondiale de la santé (OMS), le diagnostic F 45.4 est celui de "Syndrome douloureux somatoforme persistant» (plus explicite en anglais: «Pain disorder related to psychological factors»). Dans la nouvelle version du Manuel diagnostique et statistique des troubles mentaux de l'American Psychiatric Association (DSM-5), ce trouble est maintenant désigné comme «trouble à symptomatologie somatique et troubles connexes».

3 Le Tribunal fédéral considère que le trouble somatoforme douloureux rentre " implicitement » dans la notion de douleurs chroniques (cf. consid. 3.3). Le cas présent est à distinguer d'un arrêt antérieur et en partie similaire où la prise en charge par la caisse d'un autre opioïde avait été niée, notamment au motif que le médicament n'était pas prescrit dans son indication thérapeutique approuvée par Swissmedic et reprise par l'OFSP dans sa LS (cf. arrêt 9C_561/2010 du 6 juin 2011).

4 Meredith Noble et al., Long-Term Opioid Therapy for Chronic Noncancer Pain: A Systematic Review and Meta-Analysis of Efficacy and Safety, Journal of Pain and Symptom Management 35 (2), p. 214-228 (2008) ("Opioid therapy for chronic noncancer pain (CNCP) is controversial due to concerns regarding long-term efficacy and adverse events (including addiction). We systematically reviewed the clinical evidence on patients treated with opioids for CNCP for at least six months. [...] Signs of opioid addiction were reported in only $0.05 \%(1 / 2,042)$ of patients and abuse in only $0.43 \%(3 / 685) »)$.

5 Le syndrome de dépendance consiste en un «ensemble de phénomènes comportementaux, cognitifs et physiologiques survenant à la suite d'une consommation répétée d'une substance psychoactive, typiquement associés à un désir puissant de prendre la drogue, à une difficulté à contrôler la consommation, à une poursuite de la consommation malgré des conséquences nocives, à un désinvestissement progressif des autres activités et obligations au profit de la consommation de cette drogue, à une tolérance accrue, et, parfois, à un syndrome de sevrage physique.» Voir http://apps.who.int/classifications/icd10/ browse/2008/fr\#!/F10-F19.

6 Un arrêt du 16 septembre 2016 (9C_730/2015) dans une affaire «Myozyme II" avait déjà posé cette règle: lorsque le médicament a été admis dans la LS, en l'occurrence avec une «limitatio» très détaillée, la caisse ne peut en principe plus remettre en cause, à l'égard de son assuré, l'économicité et l'efficacité (du moins au début du traitement) dudit médicament (consid. 6).

7 L'assurée avait d'ailleurs reçu instruction en 2011 de son assurance-invalidité de se soumettre à un traitement de sevrage; elle n'y avait pas donné suite et sa rente-invalidité avait été supprimée, ce que le Tribunal fédéral avait confirmé dans un arrêt du 12 novembre 2013 (8C 545/2013).

8 Le droit à la protection de la bonne foi trouve son fondement dans l'art. 9 de la Constitution («Toute personne a le droit d'être traitée par les organes de l'Etat sans arbitraire et conformément aux règles de la bonne foi.»), mais est essentiellement une création - au demeurant ancienne-de la jurisprudence.

9 La médecine rencontre d'ailleurs d'importantes difficultés à identifier le ou les traitements efficaces contre les troubles somatoformes douloureux. Cf. par ex. Carlo Delli Noci, et Chantal Berna, Traumatisme et douleur chronique: échos et amplifications des souffrances physiques et psychiques, Revue médicale suisse p. 1395-99, 2015.

10 L'arrêt aurait pu préciser quel volume de prestations doit être payé par la caisse pour faire naître la confiance de l’assuré. Un arrêt de 1999 du Tribunal fédéral des assurances suggère une limite inférieure avoisinant les 3 mois (K 19/99, RAMI 1999 no. KV 97 p. 525). Un autre arrêt du 27 septembre 1993 (non publié) rappelle que le paiement par l'assurance doit être ininterrompu, de sorte que des paiements ponctuels pendant une période donnée ne font pas naître une confiance légitime quant à la prise en charge pour une autre période séparée (par ex. une année plus tard).

11 Les précédents arrêts du Tribunal fédéral appliquant le principe de la confiance dans la prise en charge de prestations par l'assurance-maladie remontent à 2015 et 2009 (arrêt 9C 5/2015 du 31 juillet 2015 et arrêt 9C_917/2007 du 14 janvier 2009; tous deux en italien). Pour sa part, le Tribunal fédéral des assurances avait tranché une poignée d'affaires mettant en œuvre le droit à la protection de la bonne foi dans les relations entre assurés et caisses (cf. arrêt K 107/05 du 25 octobre 2005, application admise; arrêt K 44/03 du 19 novembre 2004, application niée; arrêt K 25/02 du 23 septembre 2002, application admise; K 19/99 du 17 septembre 1999, application admise). 